\title{
Recent Progress on dispersion interferometers for nuclear fusion and low-temperature plasmas
}

\author{
T. Akiyama ${ }^{\mathrm{a}}$, M.A. Van Zeeland ${ }^{\mathrm{a}}$, T.N. Carlstrom ${ }^{\mathrm{a}}$, R.L. Boivin ${ }^{\mathrm{a}}$, K.J. Brunner ${ }^{\mathrm{b}}$,

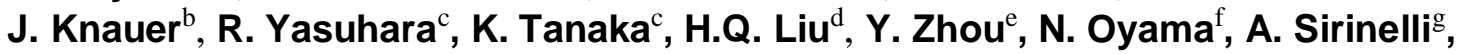 \\ K. Urabe ${ }^{\text {h }}$ N. Shirai ${ }^{\mathrm{i}}$ \\ ${ }^{a}$ General Atomics \\ P.O. Box 85608, San Diego, CA 92186-5608, USA \\ ${ }^{b}$ Max-Plank-Institute for Plasma Physics \\ Wendelsteinstraße 1, 17491 Greifswald, Germany \\ ${ }^{c}$ National Institute for Fusion Science \\ 322-6 Oroshi-cho, Toki, Gifu, 509-5292, Japan \\ ${ }^{d}$ Institute of Plasma Physics Chinese Academy of Sciences \\ 350 Shushanhu Rd., Hefei, Anhui,230032, China \\ ${ }^{e}$ Southwestern Institute of Physics \\ P.O. Box 432, Chengdu, Sichuan 610041, China \\ ${ }^{f}$ National Institutes for Quantum and Radiological Science and Technology \\ 801-1 Mukoyama, Naka-shi, Ibaraki-ken 311-0193, Japan \\ ${ }^{g}$ ITER Organization \\ Route de Vinon-sur-Verdon, CS 90 046, 13067 St. Paul-lez-Durance, France \\ ${ }^{h}$ Kyoto University \\ C3-b3S18 Kyoto-Daigaku Katsura, Nishikyo-ku, Kyoto 615-8540, Japan \\ ${ }^{i}$ Hokkaido University \\ Kita 8, Nishi 5, Kita-ku, Sapporo, Hokkaido, 060-0808 Japan \\ E-mail: akiyamatefusion.gat.com
}

ABSTRACT: A dispersion interferometer (DI) is known to be less sensitive to mechanical vibrations, which is one of the main sources of error for conventional interferometers. A simple optical configuration is also one of the advantages of the DI approach. Since the first application of a homodyne DI on a nuclear fusion plasma device in the 1990s, several interferometer techniques have improved the operation of the DI. Improvements in nonlinear crystals have also improved the performance and availability of the DI. Immunity to neutral gas density changes, which is needed for low-temperature plasma measurements, is also found. A remaining challenge is the suppression of the offset drifts, which can be significant. Recent studies confirmed that they are caused by ambient humidity changes.

KEYWORDS: dispersion interferometer, homodyne, heterodyne, phase modulation, nonlinear crystal

\footnotetext{
* Corresponding author.
} 


\section{Contents}

1. Introduction 1

2. Brief description of dispersion interferometer 2

$\begin{array}{ll}2.1 \text { Principle of dispersion interferometer } & 2\end{array}$

2.2 Comparison with a two-color interferometer 3

3. Technical Progress 4

3.1 Homodyne detection $\quad 4$

$\begin{array}{ll}3.2 \text { Phase modulation } & 6\end{array}$

$\begin{array}{ll}3.3 \text { Heterodyne detection } & 7\end{array}$

$\begin{array}{ll}3.4 \text { Combination with a polarimeter } & 8\end{array}$

$\begin{array}{lr}3.5 \text { Nonlinear crystal } & 9\end{array}$

4. Applications $\quad 10$

$\begin{array}{lr}\text { 4.1 Nuclear fusion plasmas } & 10\end{array}$

4.1.1 Homodyne detection $\quad 10$

4.1.2 Phase modulation and combination with a polarimeter 11

4.1.3 Heterodyne detection 11

4.2 Low-temperature plasmas 12

5. Remaining Challenges

6. Summary 15

\section{Introduction}

An interferometer is a powerful and general tool to measure the electron density of a plasma [1]. Since the index of refraction of a plasma depends on the electron density, the phase difference between waves which pass inside (probe wave) and outside (reference wave) of a plasma gives the line averaged electron density along the beam path in the plasma. Generally speaking, the interferometer can achieve high density resolution by selecting an appropriate light source wavelength under a well-controlled environment. This is one of the reasons why the interferometer is widely used. One of fundamental challenges of interferometers, however, is the measurement error caused by mechanical vibrations of optical components and cannot be suppressed completely by mechanical means alone. Even if the vibrations are very small, changes of the path length caused by these vibrations can be comparable to the wavelength of the probing laser. They result in a non-negligible phase shift, which is typically comparable to that caused by the probed plasma. Hence the reduction of vibration effects is a necessary issue that must be addressed to realize the desired density resolution.

Much effort has been devoted in the reductions of measurement errors due to the vibrations [1]. For example, rigid structures have been constructed to limit and isolate vibration sources. A 
vibration damping system like a pneumatic cushion also works to reduce the vibration amplitude. The dependences of the plasma-induced and vibration-induced phase shifts on the probe wavelength are different, being plasma $(\propto \lambda)$ and vibrations $(\propto 1 / \lambda)$. Hence simultaneous measurements with two different wavelengths can be used to separate the two separate phase shift contributions [1]. This so-called "two-color" interferometer is a typical configuration for fusion devices worldwide, especially using a short-wavelength (e.g. $10.6 \mu \mathrm{m})$ laser combined with a visible laser such as a $0.632 \mu \mathrm{m} \mathrm{HeNe} \mathrm{[2-6].} \mathrm{Active} \mathrm{feedback} \mathrm{alignment} \mathrm{control} \mathrm{of} \mathrm{the} \mathrm{laser} \mathrm{light}$ is also one of the solutions to minimize vibrations/motion. A prototype of toroidal interferometer polarimeter (TIP) for ITER [7] established a sophisticated control system, and confirmed that the active control improved the density resolution. Two-color interferometers are being used as a primary electron density diagnostic on high-temperature nuclear fusion plasma devices.

The other solution, and focus of this work, is a dispersion interferometer (DI) [8]. Its unique feature is smaller sensitivity to vibrations. In this approach, the measured phase shift is dominated by the plasma, and high density resolution is obtained without the need for large vibration reductions. With this advantage, the DI has been added to various fusion plasma devices worldwide. The performance of DI systems has been steadily improving by the introduction of various innovations leading to a variety of DIs which will be discussed here. In recent years, the DI has also been found to be less sensitive to changes of neutral gas density [9], a feature which makes measurements of low-temperature plasmas using conventional interferometery rather difficult. With this advantage, applications of the DI are expanding, not only in the field of nuclear fusion plasmas, but also in low-temperature plasma implementation.

This paper describes recent technological progress of the DI. Sec. 2 describes the principle of the DI, comparing with a normal two-color interferometer. Sec. 3 shows standard interferometer techniques as applied to the DI. Sec. 4 shows applications of the DI to nuclear fusion and low-temperature plasmas. Remaining challenges of the DI are described in Sec. 5.

\section{Brief description of dispersion interferometer}

\subsection{Principle of dispersion interferometer}

Figure 1 is a conceptual view of the DI. As the principle of the DI has been described elsewhere [8], only the basic principle is shown here. Laser light is injected into a nonlinear crystal to generate the second harmonic light. Although the fundamental and the second harmonic lights are drawn separately in figure 1, their paths virtually overlap. In this manner, the probe light of the DI is a mixture of these two wavelengths. After passing through a plasma, the second nonlinear crystal doubles the frequency of the fundamental light. The phases of the two second harmonic light $\varphi_{1}$ and $\varphi_{2}$ are given as follows:

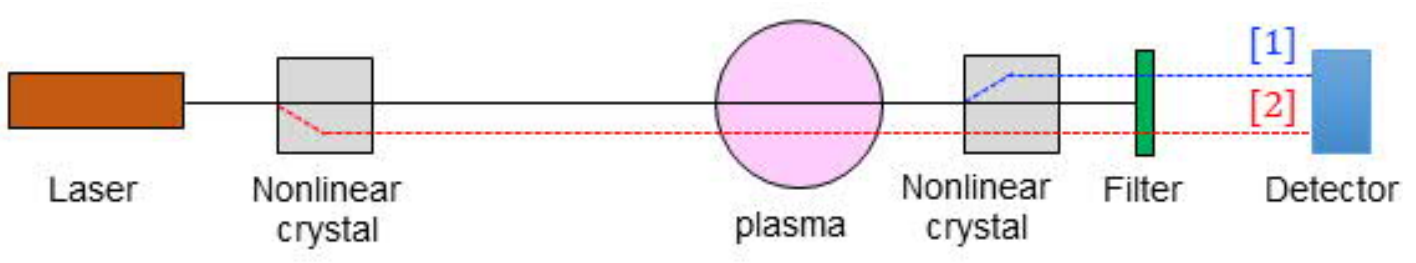

Fig. 1: Concept of a dispersion interferometer 


$$
\begin{gathered}
\text { [1]: } \varphi_{1}=2\left(\omega t+\frac{\omega n \Delta d}{c}+\frac{c_{p} \bar{n}_{e} L}{\omega}+\phi_{1}\right) \\
{[2]: \varphi_{2}=2 \omega t+\frac{2 \omega n \Delta d}{c}+\frac{c_{p} \bar{n}_{e} L}{2 \omega}+\phi_{2}} \\
c_{p}=\frac{e^{2}}{2 c \epsilon_{0} m_{e}}
\end{gathered}
$$

Here, $\omega$ is the angular frequency of the laser light, $n$ is the index of refraction of air, $\Delta d$ is the change in the path length by the vibrations, $c$ is the light speed, $\bar{n}_{e}$ is the line averaged electron density, $L$ is the path length in a plasma, $\phi_{1}$ and $\phi_{2}$ are phase offsets, $e$ is the electron charge, $\epsilon_{0}$ is the permittivity in vacuum, and $m_{e}$ is the electron mass. Remaining fundamental light is filtered out and the interference between the two second harmonic beams is detected. The phase of the interference signal is a subtraction between Eq. (1) and (2), and it becomes

$$
\begin{gathered}
I=I_{D C}+I_{A C} \cos \left(\varphi_{1}-\varphi_{2}\right)=I_{D C}+I_{A C} \cos \left(\frac{3}{2} \frac{c_{p} \bar{n}_{e} L}{\omega}+\phi_{0}\right) \\
I_{D C}=I_{1}+I_{2}, I_{A C}=2 \sqrt{I_{1} I_{2}} \\
\phi_{0}=\phi_{1}-\phi_{2} .
\end{gathered}
$$

$I_{1}$ and $I_{2}$ are detected intensities of the second harmonic beams. Since the phase shifts by the vibrations in Eq. (1) and (2) result in the same value, they are cancelled by the subtraction. This is the reason why the DI is less sensitive to vibrations. On the other hand, the phase shifts by the plasma are different due to plasma dependence on the wavelength of the index of refraction of a plasma (dispersion), the phase difference which is proportional to the electron density only remains in the interference signal.

In addition to intrinsic vibration cancellation, the DI can reduce the phase variations caused by neutral gas density [9]. This is a great advantage for the electron density measurements of lowtemperature plasmas. Low-temperature plasmas are produced in atmosphere or in low pressure conditions, and heat the neutral gas in or around the plasma. Consequently, the gas density changes, and the index of refraction of the gas $n$ also changes. The phase offset $\phi_{1}$ and $\phi_{2}$ include a phase component determined by the optical path length in the gas $\omega n L_{\text {gas }} / c$ and $2 \omega n L_{\text {gas }} / c$, respectively. Here, $L_{\text {gas }}$ is the path length in the gas. While changes of $n$ vary $\varphi_{1}$ and $\varphi_{2}$, the phase variations are almost common between $\varphi_{1}$ and $\varphi_{2}$. Hence the DI can significantly reduce the phase variations by the gas density changes in the same way as $\Delta d$.

\subsection{Comparison with a two-color interferometer}

As shown in Sec. 2.1, the probe light of the DI is a mixture of the fundamental and second harmonic light. Since it uses two different wavelengths, it can be roughly categorized as a twocolor interferometer.

A traditional two-color vibration compensated interferometer, however, is equipped with two laser sources with different wavelengths, frequency shifters for the different wavelengths, detectors and beam combiners/splitters for the two wavelengths. It measures the phase difference including contributions from the plasma and vibration components for each wavelength, and resolves the phase differences afterward by solving simultaneous equations [1]. The DI needs only one laser source and one detector. The "second color" is generated with a nonlinear crystal before entering the plasma. The "first color", the fundamental component, which passes through a plasma, is converted into the second harmonic component by a frequency-doubling second nonlinear crystal. A single interference signal between second harmonic components, which are 
generated before and after plasma, is detected. The plasma-induced phase shift using the first color remains in one of the second colors. In this process, the vibration induced phase shift is cancelled automatically as described in Sec. 2.1, and no further signal analysis is required to remove vibration.

Generation of the second color with the nonlinear crystal also provides additional benefits. One is a simpler interferometer configuration, this would be the case even if used as a traditional two-color interferometer. The DI needs a single laser source and single detector. Also, as one of the wavelength components corresponds to a reference in the conventional interferometer, no dedicated reference path is necessary for the DI. The other benefit is a good overlapping of the two wavelengths. As found in the TIP prototype [7], accuracy of overlapping of the two laser colors can determine the degree of vibration cancellation. Generally speaking, the second harmonic component well overlaps the fundamental component.

Requirements for the laser source are also different between the two-color interferometer and the DI. For a two-color interferometer based on a $\mathrm{CO}_{2}$ laser, laser power of the order of $1 \mathrm{~mW}$ at a detector is normally sufficient. On the DI, second harmonic generation (SHG) needs a high power, preferably larger than hundreds of milliwatts at least. In case of a long beam transmission, the laser power can be attenuated. Considering the SHG after the beam attenuation along the beam transmission, the DI requires a high power $(\sim 50 \mathrm{~W})$ laser source. Instead, a less coherent laser will be available for the DI. This is because the two second harmonics to be interfered have almost the same beam path and laser oscillation noises are cancelled. On the two-color interferometer, path lengths of the plasma measurement and reference leg are typically made very nearly the same for good interference and cancellation of frequency noise from the laser oscillation. The DI intrinsically cancels this frequency noise.

\section{Technical Progress}

Starting with a conventional homodyne interferometer, some interferometer configurations have been introduced to the DI in last two decades. Each configuration has unique characteristics, and hence users can choose an appropriate interferometer configuration which is suitable for their application. Together with the configuration, optical components for the DI have improved. In the sections below, characteristic advantages/disadvantages of each configuration are described. As for the hardware, progress of a nonlinear crystal will be shown as it is a key component of the DI.

\subsection{Homodyne detection}

The DI was first introduced on a magnetically confined nuclear fusion plasma device in the 1990s. It essentially corresponded to a homodyne interferometer [1] as shown in Fig. 1 and Fig. 2(a). The normal homodyne interferometer uses two (probe and reference) laser beams with the same frequency and with the different beam paths (inside and outside plasma). On the other hand, the DI uses the two laser beams with different frequencies (fundamental and the second harmonic) and with the same beam path. Although there are such differences in the configuration, the resulting interference signal of the DI Eq. (5) is the same as that of the homodyne interferometer except the coefficient of the proportionality coefficient to the electron density. In order to extract the phase, the homodyne detection needs $I_{D C}$ and $I_{A C}$ in Eq. (5). In practice, two detectors are used as shown in Fig. 2(b). Here, polarizations of second harmonic components generated before 


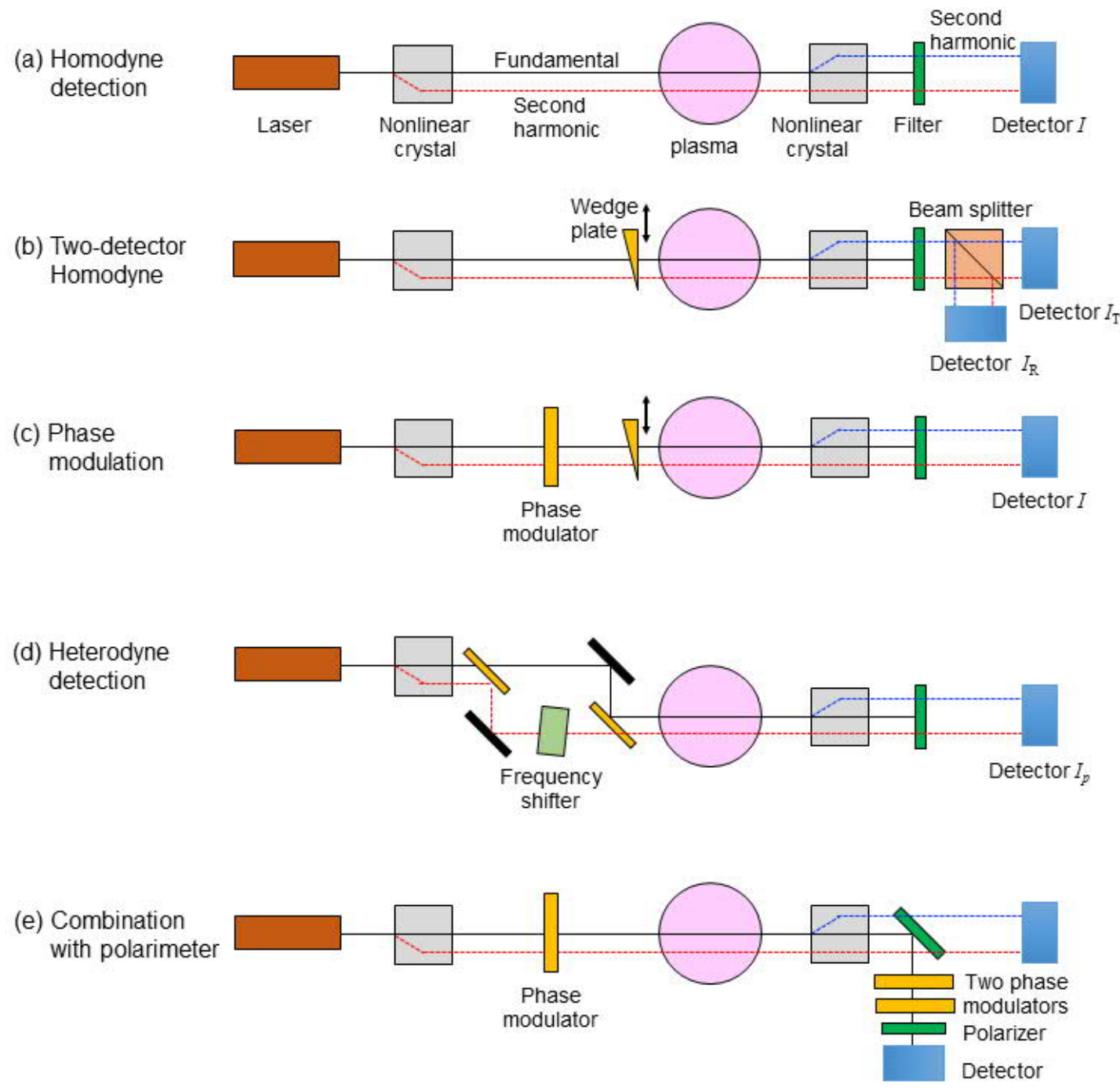

Fig. 2: Detection techniques of a dispersion interferometer.

and after a plasma are made orthogonal by use of type-II nonlinear crystals [8] or a dual wavelength wave plate [10]. Setting the axis of a polarization beam splitter at $45^{\circ}$ with respect to the polarization of the second harmonics, the detected two interference signals $I_{+}$and $I_{-}$are

$$
\begin{aligned}
& I_{+}=\alpha_{+} A_{+}\left\{I_{D C}+I_{A C} \cos \left(\phi_{p}+\phi_{0}\right)\right\} \\
& I_{-}=\alpha_{-} A_{-}\left\{I_{D C}-I_{A C} \cos \left(\phi_{p}+\phi_{0}\right)\right\}
\end{aligned}
$$

where $\alpha_{+}$and $\alpha_{-}$are constants determined by the detector responsivity, $A_{+}$and $A_{-}$are the amplifier gains, $\phi_{p}$ is the phase shift caused by a plasma $[8,10]$. Note that the phase of the one of the measured interference signal is shifted by $\pi$. Making the DC components the same by adjusting the amplifier gains, the difference of the two interference signals becomes

$$
I_{+}-I_{-}=2 \alpha_{+} A_{+} I_{A C} \cos \left(\phi_{p}+\phi_{0}\right) \text {. }
$$

The amplitude of the cosine wave is evaluated separately. By changing $\phi_{0}$ larger than $\pi$ (movable a wedge plate or rotating a plate, instead of a plasma), the amplitude can be obtained. This is the same process conducted for the normal homodyne interferometer. The phase shift $\phi_{p}$ is evaluated as below. 


$$
\phi_{p}=\cos ^{-1}\left(\frac{I_{+}-I_{-}}{2 \alpha_{+} A_{+} I_{A C}}\right)-\phi_{0}
$$

In reality, $I_{A C}$ can vary during a plasma discharge due to, for example, laser instabilities and refraction of the probe light in a plasma. The intensity variations lead to uncertainties in the evaluated phase.

The DI on field-reversed configuration (FRC) plasma device in UC Irvine (UCI) [10] utilizes $I_{+}+I_{-}$in addition to $I_{+}-I_{-}$. The ratio $R$ is defined as

$$
\begin{gathered}
R=\frac{I_{+}-I_{-}}{I_{+}+I_{-}} \approx V \sin \left(\phi_{p}+\phi_{0}\right) \\
V=\frac{2 \sqrt{\beta_{1} \beta_{2}}}{\beta_{1}+\beta_{2}} \quad(12)
\end{gathered}
$$

where $\beta_{1}, \beta_{2}$ are efficiencies of SHG before and after a plasma. Use of the ratio improves the effects of the intensity variations. While $V$ is also determined by the same calibration without a plasma, it is reported that $V$ value had been maintained for several tens of days, and demonstrated sufficient long term stability. In case of a relatively large interferometer system, decrease of $\beta_{2}$ during a shot, which can be caused by vibrations or refraction in a plasma should be noted.

The range of the phase shift is limited below $\pi$ to determine the electron density without ambiguity. After the phase shift exceeds $\pi$, changes of $R$ cannot tell whether the density increased or decreased. Since the phase shift is proportional to the wavelength, a shorter wavelength is preferable. In a normal interferometer, the shorter wavelength also increases the phase shift by the mechanical vibrations $2 \pi n \Delta d / \lambda$. Hence the density resolution of the shorter wavelength interferometer tends to be worse. In contrast, the DI is less sensitive to mechanical vibration, deterioration of the density resolution is smaller. DIs on GDT [8] and on FRC [10] uses a Nd:YAG laser whose wavelength is $1.064 \mu \mathrm{m}$, and it covers the required electron density range.

This configuration is quite simple, and a total optical system can be more compact by using optical fibers [10]. Since this method does not utilize any modulators, the time response is determined by a bandwidth of detection components and sampling frequency.

\subsection{Phase modulation}

The phase modulation technique was originally introduced to the DI on TEXTOR [11]. This configuration enables reduction of measurement errors caused by the intensity variations even during a shot.

The arrangement of this configuration is shown in Fig. 2 (c). This configuration uses a typeI nonlinear crystal, so that the polarization of the second harmonic light is perpendicular to that of the fundamental one. A phase modulation of $\rho \sin \left(\omega_{m} t\right)$ is added to either fundamental or second harmonic light. Here, $\rho$ is the retardation, $\omega_{m}$ is the angular frequency of the drive signal of the phase modulator. Adding the phase modulation to the second harmonic light, the resultant interference signal Eq. (4) is modified as below.

$$
I=I_{D C}+I_{A C} \cos \left(\rho \sin \omega_{m} t+\phi_{p}+\phi_{0}\right)
$$

When $\rho$ is set at larger than $\pi$, the phase in Eq. (13) changes more than $2 \pi$ in one modulation cycle. Supposing that the phase shift does not change during the one cycle $2 \pi / \omega_{m}, I_{D C}$ and $I_{A C}$ can be evaluated even during a plasma shot. When $\omega_{m} t=N \pi(N$ : integer), which satisfies $\sin \omega_{m} t=0$, the phase difference due to the plasma can be obtained.

$$
I\left(\omega_{m} t=N \pi\right)=I_{D C}^{e v a}+I_{A C}^{e v a} \cos \left(\phi_{p}+\phi_{0}\right)
$$




$$
\therefore \phi_{p}\left(\omega_{m} t=N \pi\right)=\cos ^{-1}\left(\frac{I-I_{D C}^{e v a}}{I_{A C}^{e v a}}\right)-\phi_{0}
$$

Here, $I_{D C}^{e v a}, I_{A C}^{e v a}$ are evaluated intensities in real time by the above process. This signal process can be conducted with field-programmable gate arrays (FPGAs) [12]. The drive frequency $\omega_{m}$ should be higher than the frequency or the time scale of electron density changes to be measured.

Additional methods to extract the phase difference from the modulated interference signal also exist. Some DIs [13-18] evaluate the phase difference from a ratio of amplitudes of fundamental and second harmonic components of the drive frequency $\omega_{m}$. Eq. (13) consists of harmonics of the drive frequency, and the amplitudes of the fundamental and the second harmonics $I_{\omega_{m}}, I_{2 \omega_{m}}$ are expressed as follows.

$$
I_{\omega_{m}}=-2 I_{A C} J_{1}(\rho) \sin \left(\phi_{p}+\phi_{0}\right), \quad I_{2 \omega_{m}}=2 I_{A C} J_{2}(\rho) \cos \left(\phi_{p}+\phi_{0}\right)
$$

Here, $J_{1}(\rho)$ and $J_{2}(\rho)$ are the Bessel functions of first and second order. Setting the $\rho$ at $2.63 \mathrm{rad}$., which satisfies $J_{1}(\rho)=J_{2}(\rho)$, the phase difference can be evaluated as below expression.

$$
\phi_{p}=\tan ^{-1}\left(\frac{J_{2}(\rho)}{J_{1}(\rho)} \frac{I_{\omega_{m}}}{I_{2 \omega_{m}}}\right)-\phi_{0}=\tan ^{-1}\left(\frac{I_{\omega_{m}}}{I_{2 \omega_{m}}}\right)-\phi_{0}
$$

This is a type of the quadrature detection from the viewpoint of generating sine and cosine terms. This scheme allows the evaluation of the phase difference without $I_{D C}$ and $I_{A C}$. Hence the measurement errors attributable to the intensities are intrinsically smaller. The amplitudes of the harmonic components Eq. (16) are extracted with lock-in amplifiers [13], and signal processing is easily conducted without any circuit developments. In this method, the drive frequency of the modulator and resulting time constant of the density measurement are determined by the frequency bandwidth of the lock-in amplifier. A software lock-in technique [19] has more flexibility and extendibility. A similar, but more sophisticated algorithm for quadrature generation is being used on W7-X [16]. It does not need Fast Fourier Transform and can be easily implemented by a FPGA. On the other hand, this scheme needs a calibration using a wedge plate. This is necessary, since the analytical retardation value $\rho$ to meet the $J_{1}(\rho)=J_{2}(\rho)$ condition (or in the W7-X case $H_{0}(\rho)=J_{0}(\rho)$, where $H_{0}$ is the Struve function of zeroth order) is modified by the physical setup of the system, e.g. off-axis passes through the modulator. When the actual retardation value is not appropriate, the measured phase difference becomes different from the actual phase difference. This is the same reason for the calibration conducted in a polarimeter [20]. While fine adjustment of the retardation can minimize the nonlinearity, conducting the calibration is preferable.

There are two available phase modulators. One is an electro-optic modulator (EOM), the other is a photoelastic modulator (PEM). Since the PEM is a resonant modulator, whose drive frequency is determined by the physical crystal size, noise frequencies are hard to be contained in the modulation frequency. However, the drive frequency is limited to less than $100 \mathrm{kHz}$, which limits the time resolution. In contrast, the frequency of the EOM can be higher than $1 \mathrm{MHz}$. Although there is no comprehensive comparison between two phase modulators with the same condition, the PEM and the EOM will be quantitatively suitable for the density monitor with a good density resolution and fluctuation measurements with a wide bandwidth, respectively.

\subsection{Heterodyne detection}

Most present two-color interferometers utilize a heterodyne detection scheme. As in standard heterodyne interferometers, the heterodyne DI is free from intensity variations to evaluate the phase difference. Different from other DI approaches, the heterodyne DI does not need 
calibration. This DI scheme was first introduced on DIII-D [21, 22]. The frequency shift for heterodyne detection is typically tens of $\mathrm{MHz}$, and hence the bandwidth is wide enough even for measuring density fluctuations in $\mathrm{MHz}$ range.

The conceptual diagram of the heterodyne DI is shown in Fig. 2 (d). Different from other DI configurations, the overlapping fundamental and the second harmonic light are physically separated first. The fundamental and the second harmonic have different paths, and one of the colors is shifted by $\omega_{m}$ with an Acousto-Optic (AO) cell. After the frequency shift, the two colors are combined again and the overlapped beams are injected into a plasma, as with the other DIs. The detected interference signal $I_{p}$ is

$$
I^{p}=I_{D C}^{p}+I_{A C}^{p} \cos \left(\omega_{m} t+\phi_{p}+\phi_{0}\right)
$$

The drive signal of the AO cell is available as a reference signal $I^{r}$

$$
I^{r}=I_{A C}^{r} \cos \left(\omega_{m} t\right) .
$$

The phase difference between $I^{p}$ and $I^{r}$ can be evaluated with the same methods used for the normal heterodyne interferometers: IQ detector [6], a phase counter [23], and digital phase demodulator [7, 24].

The degree of the vibration cancellation depends on the accuracy of the overlapping of the fundamental and the second harmonic light. The heterodyne DI requires manual alignment after the frequency shift, and careful alignment is essential. This is the same as the requirement for a two-color interferometer [2]. Vibrations along the path where the two wavelength are not aligned cannot be cancelled, so that part of the frequency shift optics should be made as compact (and robust) as possible. An optical reference instead of the AO cell driver can be obtained by splitting the probe light into two after recombination of two wavelengths and sending to a separate crystal/detector combination. Even if the phase is perturbed within the frequency shift optics, the additional phase shift is shared in both the reference and the probe. Hence, the additional phase shift will be cancelled.

\subsection{Combination with a polarimeter}

A polarimeter, which measures the Faraday rotation [1], can be used for the electron density measurements. The Faraday rotation is the rotation of the plane of the polarization and is the rotation angle $\alpha$ is given by the following expression.

$$
\begin{gathered}
\alpha=\frac{e}{2 c m_{e} n_{c}} \int n_{e} \vec{B} \cdot d \vec{l} \\
n_{c}=\frac{\omega^{2} \varepsilon_{0} m_{e}}{e^{2}}
\end{gathered}
$$

where $n_{c}$ is the cut-off density for a wave with an angular frequency of $\omega, \vec{B}$ is the magnetic field vector, $\vec{l}$ is the direction of the wave propagation. Assuming some magnetic field information, approximate line-integrated electron density can be evaluated from the Faraday rotation angle. The density resolution of interferometers (including the DI) is relatively high, but the interferometers can encounter a fringe counting failure (called a "fringe jump") because the phase has $2 \pi$ ambiguity. The electron density is proportional to the total phase shift, which is typically larger than $2 \pi$ (one fringe). Hence the time history of the phase is necessary to evaluate the electron density. If the phase measurement is accidentally interrupted, the total phase shift could be lost. This is one of the main drawbacks of all interferometer schemes. The Faraday rotation 
angle is less than $2 \pi$ by choosing an appropriate wavelength, and hence the instantaneously measured rotation angle can give the line-integrated electron density.

In DIs, the fundamental component is usually dumped after the SHG after passing through a plasma. The fundamental component, however, is available for the measurement of the Faraday rotation angle. A benefit of the combination of the interferometer and polarimeter, which share a probe beam, is to increase reliability of the electron density measurement. A combined polarimeter can help in correcting any fringe jump of the interferometer.

Fig. 2 (e) shows one of the concepts of the combination of the DI and the polarimeter. "Dual PEM" polarimeter [25] has a good compatibility with the DI. The fundamental component is sent to a pair of PEMs with different drive frequencies $\omega_{m 1}$ and $\omega_{m 2}$. The detected intensity following a polarizer is a summation of harmonics of the drive frequencies, and the amplitudes $I_{2 \omega_{m 1}}$ and $I_{2 \omega_{m 2}}$ of second harmonics of the each drive frequency are:

$$
\begin{gathered}
I_{2 \omega_{m 1}}=-\left(I_{0} / \sqrt{2}\right) J_{2}\left(\rho_{1}\right) \cos (2 \alpha) \\
I_{2 \omega_{m 2}}=\left(I_{0} / \sqrt{2}\right) J_{2}\left(\rho_{2}\right) \sin (2 \alpha) .
\end{gathered}
$$

The Faraday rotation angle $\alpha$ is obtained from the ratio of these amplitude.

$$
\alpha=-\frac{1}{2} \tan ^{-1}\left(\frac{I_{2 \omega_{m 2}}}{I_{2 \omega_{m 1}}}\right)
$$

By selecting different frequencies $\omega_{m}, \omega_{m 1}, \omega_{m 2}$, the DI and the polarimeter do not affect each other and are completely independent. The individual performance of each diagnostic is unaffected by this combination.

\subsection{Nonlinear crystal}

A high-quality nonlinear crystal, which is used for frequency doubling, is a critical component in the DI. Available laser sources for the DI is determined by the wavelength range of the nonlinear crystal. At present, second harmonics with a sufficient power for diagnostic purposes can be generated from 1 to $10 \mu \mathrm{m}$ by commercial nonlinear crystals. Hence a continuous-wave (cw) $\mathrm{CO}_{2}$ laser or a cw Nd:YAG laser have been used for the DI.

The efficiency of the SHG $\eta$ is given by [25]

$$
\begin{gathered}
\eta=2\left(\frac{\mu}{\varepsilon_{0}}\right)^{2} \frac{\omega^{2} d_{e f f}^{2} l^{2}}{n^{3}}\left(\frac{P_{\omega}}{\pi w_{0}^{2}}\right)\left\{\frac{\sin (\Delta k l / 2)}{\Delta k l / 2}\right\} \\
\Delta k=k_{2 \omega}-2 k_{\omega}
\end{gathered}
$$

where $d_{\text {eff }}$ is the effective nonlinearity, $l$ is the length of the crystal, $n$ is the index of refraction of the nonlinear material at fundamental wavelength, $w_{0}$ is the beam waist ( $1 / \mathrm{e}^{2}$ power radius), and $k_{2 \omega}$ and $k_{\omega}$ are wavenumbers of the second harmonic and the fundamental components, respectively. The last term, which includes $\Delta k$, stands for the phase matching condition of the crystal. Normally, $\Delta k$ should be zero. Since the efficiency is proportional to the power density, the efficiency of SHG by a cw laser used for the DIs is generally much smaller than that by a pulsed laser. Hence optimization of SHG is indispensable to have a sufficient signal to noise ratio of the detected interference signal.

There are two types of frequency doublers to satisfy the phase matching condition $\Delta k=0$. If the phases of two components $k_{2 \omega}$ and $2 k_{\omega}$ mismatch in the crystal, the second harmonic components generated at each position can weaken each other, and the efficiency becomes low. For the phase matching, one uses conventional birefringence of a nonlinear material, the other uses the periodic domain inversion structure. For $10 \mu \mathrm{m}$ wavelength, the former used for the DIs are $\mathrm{ZnGeP}_{2}$ 
[11], $\mathrm{AgGaSe}_{2}$ [13-18], and AgGaS the latter is OP-GaAs [21-22, 27-28]. One of the advantages of former is that they are easy to use and suitable for use at room temperature. Relatively large area of an incident plane, larger than $5 \times 5 \mathrm{~mm}$, make beam alignment and a laser focusing condition easier. Although $\mathrm{ZnGeP}_{2}$ has rather high nonlinearity and high damage threshold among the frequency doublers, its absorption around $10 \mu \mathrm{m}$ is also high [30]. The nonlinearity and the damage threshold of $\mathrm{AgGaSe}_{2}$ are lower than $\mathrm{ZnGeP}_{2}$, the absorption is small [30], and hence it has been used on several DIs [13-18]. The OP-GaAs became commercially available for infrared region in recent years. Generally speaking, the crystals with the periodic domain inversion structure exhibits higher nonlinearity, and longer crystal lengths can be manufactured, which results in higher efficiency. This is the reason why the OP-GaAs is used for the heterodyne DI, which requires the higher second harmonic power for beam alignment discussed in Sec. 3.3. Considering power loss along the long beam path such as $100 \mathrm{~m}$ on DIII-D [22] and ITER [28], the higher efficiency is preferable for the heterodyne DI. Therefore, OP-GaAs is presently the best candidate for the phase-modulated DI on ITER. On the other hand, temperature control by a heating or a cooling system is necessary for phase matching. The incident plane of presently available devices is rather thin, around $1 \times 5 \mathrm{~mm}$, and the severe beam alignment and focusing condition is required. An active feedback control of the probe beam will be indispensable to maintain the SHG efficiency if the beam displacement by the vibrations is not negligible.

\section{Applications}

\subsection{Nuclear fusion plasmas}

In nuclear fusion application, the resolution is determined by various factors like beam transmission lengths, ambient conditions, amplitudes and frequencies of mechanical vibrations, performance of optical components such as laser sources, detectors, and modulators. Definitions of the resolution may also be different in each application. In order to compare the relative merits rigorously among the detection techniques, diagnostic conditions have to be normalized. Hence the given resolutions should be seen as achieved ones in the specific conditions.

\subsubsection{Homodyne detection}

The DIs implemented on high temperature plasma devices started with homodyne detection with two detectors [8], which is still effective as a compact and fast DI. As described in the DI on the FRC on UCI [10], the optical components can be compactly modularized together with fibers, and can be installed easily without large space for optics and beam transmission line. A sensitivity of few $10^{14} \mathrm{~cm}^{-2}$ in measuring line integrated electron density is demonstrated with a time resolution of a few microseconds [10]. Time resolution can be easily increased by an increasing the sampling frequency only. Hence the homodyne detection DI could measure a time evolution of the electron density of a plasma shorter than $100 \mu \mathrm{s}$.

A challenge with this approach is how to suppress the variations of the signal magnitude $V$ in Eq (11) during a plasma discharge. The variations of $V$ can occur by changing the SHG efficiency due to beam refraction in a plasma or vibrations. Making a free beam transmission as short as possible with a short wavelength of $1.064 \mu \mathrm{m}$ whose reflection angle in a plasma is small, will improve this issue. 


\subsubsection{Phase modulation and combination with a polarimeter}

The phase modulated DI has been used on several torus devices: TEXTOR [11,12], LHD [13,14], W7-X [15,16], HL-2A [17], EAST [18]. On TEXTOR and LHD, sufficient electron density and time resolution for the density monitoring and study of MHD instabilities was achieved, and feasibility of the DIs on fusion devices was demonstrated. The DI on TEXTOR was upgraded to a four-channel system [30], modularizing each channel for easy and independent maintenance. The DI on LHD was based on initially on a PEM for phase modulation because a commercial EOM for continuous modulation in the infrared region is not widely available. The bandwidth of the DI with the PEM (drive frequency of $50 \mathrm{kHz}$ on LHD) is lower than that with the EOM (250 kHz on TEXTOR). Density measurements during pellet injections, with a time scale of approximately $1 \mathrm{~ms}$, was successfully conducted without fringe jumps [14]. The DI on W7-X is a primary electron density diagnostic and is used for real-time density control [16]. The PEM is utilized on W7-X, HL-2A, and EAST, while each system has been developing techniques for phase extraction from the interference signal Eq. (13). Achieved resolutions are reported with different definitions in the above references. As one example, random drifts in a longer time scale than $0.1 \mathrm{~s}$ limit the density resolution on LHD. The widths of the variations are $\pm 2.0 \times 10^{17} \mathrm{~m}^{-3}$ and $\pm 2.5 \times 10^{17} \mathrm{~m}^{-3}$ (corresponding to line-integrated density resolutions of $\pm 6.6 \times 10^{17} \mathrm{~m}^{-2}$ and $\pm 8.2 \times 10^{17} \mathrm{~m}^{-2}$, supposing that a path length in a plasma is $3.28 \mathrm{~m}$ ) for $3 \mathrm{~s}$ and $30 \mathrm{~min}$ measurements, respectively on LHD [14]. The response time determined by lock-in amplifiers, which are used for evaluation of the amplitudes Eq. (16), is $100 \mu$ s [33].

For redundancy of the electron density measurement on ITER, two independent density diagnostics are planned; one is the TIP [7], the other is the DI [32,29]. Having accumulated experience on various devices, the phase modulated DI was selected for the DI on ITER. It will be combined with a polarimeter shown in Fig. 2 (e). As discussed in Sec. 3.4, the combined polarimeter part is independent from the DI and is basically the same as the stand-alone PEM polarimeter [25]. The combined approach has not been fully demonstrated on a fusion device. Similar to ITER, a DI [33] is planned to be installed on JT-60SA in addition to a toroidal interferometer polarimeter [34]. Since the access is limited, the DI system will be made as compact as possible, using an air-cooled low power laser with high efficiency doubler OP-GaAs [33].

All laser sources used for these phase modulated DIs are $\mathrm{CO}_{2}$ lasers, which is optimum for the electron density and plasma size of current magnetic confined fusion devices. With an outlook toward future fusion reactors, whose plasma size is several times larger than current ones, a phase modulated DI with a cw Nd:YAG laser is being developed [35].

\subsubsection{Heterodyne detection}

For the purpose of demonstration of wide bandwidth density measurement with the DI, a heterodyne DI was developed on DIII-D [22]. As discussed in Sec. 3.3, the most important key issue is accurate overlapping of the fundamental and the second harmonic light after the AO cell. In the DI on DIII-D, a high efficiency nonlinear crystal OP-GaAs and $\mathrm{CO}_{2}$ laser (an output power of $27 \mathrm{~W}$ ) generates the second harmonic light with a power of an order of $\mathrm{mW}$. In that application, an infrared camera proved to be a powerful tool to align and monitor beam overlapping. Since the second harmonic power is usually too small to find the beam spot, visualization of the beam spot is a key issue for achieving a good resolution. Total beam transmission length was approximately $100 \mathrm{~m}$, which is comparable to the interferometers on ITER. Regardless of such a long beam transmission, alignment with the IR camera enables sufficiently good overlapping. As 
a result, vibration phase shifts were well cancelled even at the startup of the ohmic solenoid coil and when large vibrations occur [22]. The reported line-integrated density resolution is $9 \times 10^{17}$ $\mathrm{m}^{-2}$, defined as the standard deviation over a $1 \mathrm{~s}$ interval. This is a similar density resolution to that of the two-color interferometer, and density fluctuations were successfully measured [22]. Since the incident plane of the OP-GaAs is $1 \times 5 \mathrm{~mm}^{2}$ only, a feedback alignment control of the returned probe light was indispensable. Even small vibrations result in a large displacement at the second nonlinear crystal position in the case of long beam transmission. The laser light can be clipped by the thin crystal edge, and the efficiency of the SHG decreases. The feedback alignment system, which consists of a piezo tip-tilt mirror and a laser position sensor [7], was used to maintain the efficiency of the SHG on DIII-D [22].

\subsection{Low-temperature plasmas}

Immunity to the gas density discussed in Sec. 2.2 is beneficial for diagnostics of lowtemperature plasma, especially high-pressure plasmas. In cases of atmospheric pressure plasmas, which is applied to various usages like medicine, industry, environment and so on, the phase shift caused by the gas density change is significantly larger than that by a plasma complicating the evaluation of electron density with an interferometer. For example, it is reported that the phase shift by the gas density change is at least one order of magnitude larger than that by their plasma [36]. In principle, the two-color interferometer can resolve the phase shifts similar to that on the high-temperature plasma devices and a DI is not necessary to deal with the huge phase shift by the gas. A DI, however does simplify the measurement. The huge phase shift (large number of fringes) increases the risk of fringe jumps. Also, the signal to noise ratio of the phase shift by the plasma, which is much smaller than that by the gas, will become smaller because the dynamic range of the phase measurement will be determined by the total phase shift.

Urabe [9] conducted a proof of principle experiment of the electron density measurements of atmospheric pressure plasmas with the phase modulated DI. A plasma pulse duration of $1 \mathrm{~ms}$ with a repetition of $100 \mathrm{~Hz}$ was used. Since the plasma has a good reproducibility, 4096 discharges are accumulated to improve the signal to noise ratio. As a result, a temporal resolution of $110 \mu$ s and a sensitivity of the line-integrated electron density of $7 \times 10^{11} \mathrm{~cm}^{-2}$ are reported. The phase shift by the gas was also significantly reduced. The evaluated electron density by the DI was about $1 \times 10^{20} \mathrm{~m}^{-3}$ (supposing that the plasma diameter is $0.7 \mathrm{~mm}$ ) and showed a good agreement with Thomson scattering measurements [37].

Since a time resolution of $100 \mu$ s of the phase modulated DI is not sufficient for present lowtemperature plasma applications, a next step is the improvement of its time resolution. To avoid damage to organic tissues exposed to a plasma, the plasma and gas temperature have been reduced as low as possible. One of the ways to suppress the temperature increase is a short-pulse plasma operation, for example less than $1 \mu$ s [38]. Before the temperatures increase, the current from the electrode is terminated. The heterodyne DI, whose drive frequency of the AO cell is from several tens to a hundred $\mathrm{MHz}$, is capable of resolving such short pulse plasmas.

\section{Remaining Challenges}

Dispersion Interferometers have matured over the last two decades, and sufficient density resolution for density control and physics studies has been achieved on several nuclear fusion 
plasma devices worldwide. Although it is not a serious problem in current short pulse fusion plasmas with durations of a few seconds, drifts of the zero-offset discussed should be improved.

One of the primary reasons for drifts in the wavelength range of $10 \mu \mathrm{m}$ is variations of water vapor density / humidity along the laser path. The DI measures the phase shift which arises from the dependence of the index of refraction on the wavelength. Although the index of refraction of neutral gas has proportionally small dependence on the wavelength, it is not negligible. Therefore, the phase shift due to dispersion of gas remains in the interference signal together with that due to a plasma. When gas is static, the gas component is just a constant $\phi_{0}$ in Eq. (4). The dispersion component, however, has also dependence on the temperature, the pressure, and the density of the water vapor [39]. Hence variations of ambient conditions along the beam path result in the variations of the $\phi_{0}$ [7], which appears as the zero-offset drifts. Not only for the DI, this is a common challenge for two-color interferometers.

The index of refraction of air in infrared region can be given by a Mathar's fitting model as a function of the wavelength, the relative humidity $H$, the temperature $T$, and the pressure $p$ [39]. Figure 3 shows dependence of the index of refraction at 10.6 and $5.3 \mu \mathrm{m}$ and the resultant phase difference $\phi_{0}$ on the humidity.

$$
\phi_{0}=2 \frac{2 \pi\left\{n_{5.3}(T, H, p)-n_{10.6}(T, H, p)\right\} L}{\lambda}
$$

Here, $\phi_{0}$ is set at zero at $60 \%$ in Fig. 3 . The path length between two nonlinear crystals is supposed to be $7.2 \mathrm{~m}$, considering the below bench top experiment. Note that the fitting model for $4.35<\lambda<5.2 \mu \mathrm{m}$ in the Mathar's fitting model [39] is extrapolated to $5.3 \mu \mathrm{m}$ in this calculation. Since there are numerous and large electromagnetic line transitions due to $\mathrm{H}_{2} \mathrm{O}$ between 5.2 and $7.5 \mu \mathrm{m}$, that wavelength region is excluded in the fitting analyses. The predicted dependence of $\phi_{0}$ on the humidity from Fig. 3 is $\Delta \phi_{0}=0.568 \mathrm{deg} . / \% / \mathrm{m}$ at $25^{\circ} \mathrm{C}$.

Figure 4 is a photo of a bench top experiment to validate the dependence of the zero-offset drift on the water vapor density. The DI used is the phase-modulated type and the wavelength is 10.6 $\mu \mathrm{m}$. At $t=2000 \mathrm{~s}$, a humidifier starts working and increases the water vapor density by $18 \%$, maintaining the temperature constant as shown in Fig. 5 (a). After turning off the humidifier at $t$ $=3500 \mathrm{~s}$, the water vapor density gradually decreases. Air outside the laser room was introduced at $t=5900 \mathrm{~s}$. A hygrometer/thermistor was placed almost the nearest position from the humidifier to the laser path where two wavelengths were mixed (after the second nonlinear crystal, the effects of gas are completely cancelled). Since the water vapor density is not be uniform along the beam path, the measured water vapor density was the maximum value along the beam path. The measured phase shift, which is the zero-offset drift, started to increase in accordance with the 

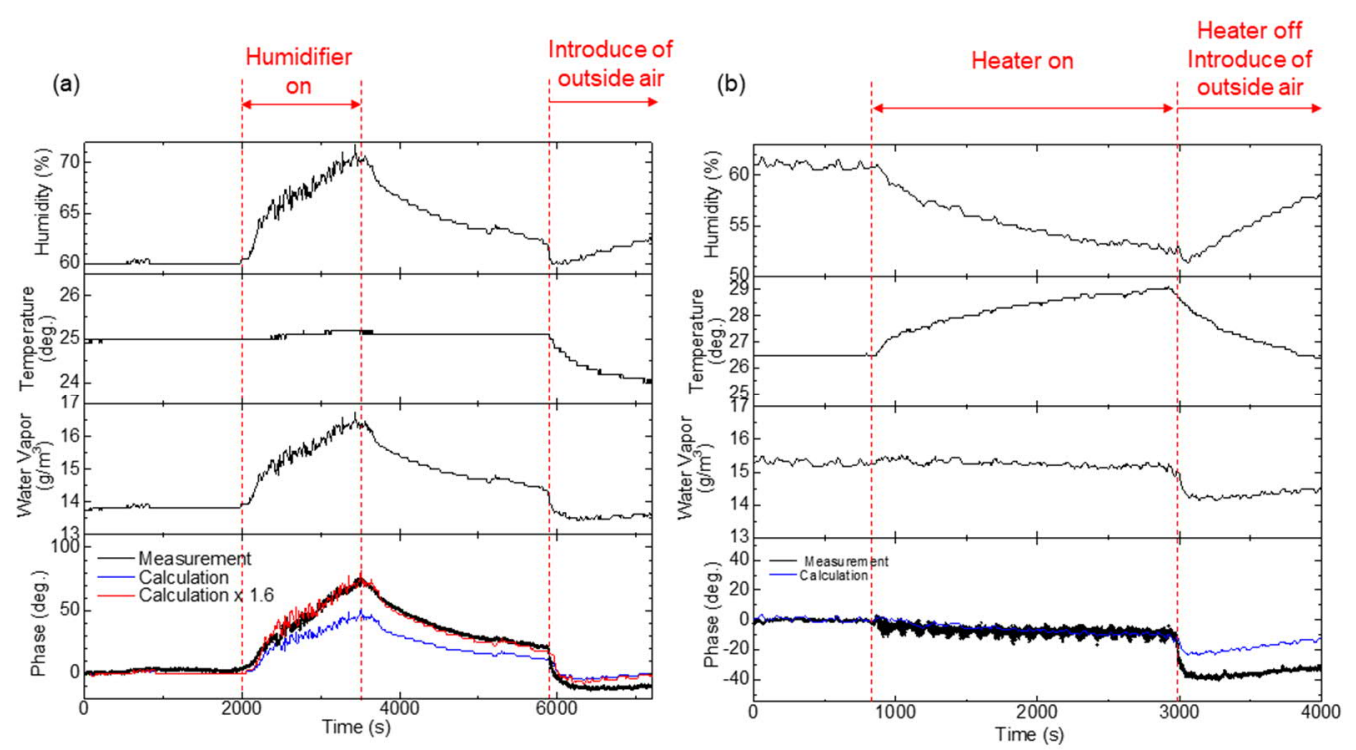

Fig. 5: Response of the phase shift to (a) the humidity and (b) the temperature changes.

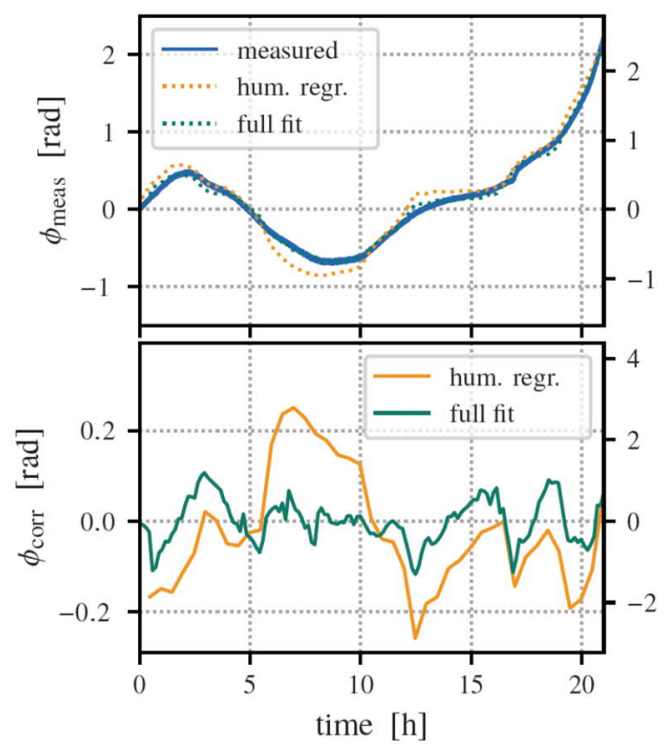

Fig. 6: Phase drift in relation to environmental parameters in around the W7-X IEDDI system [16]

increase of the water vapor density. In the bottom of the Fig. 5 (a), the calculated phase shift from the Mathar's fitting model [39] is over laid. Although the temporal behavior is well reproduced, there is a discrepancy of a factor of 1.6. Here, the water vapor density is supposed to increase uniformly along the beam path length between two nonlinear crystals $7.2 \mathrm{~m}$. This would be an overestimation of the integration of the water vapor density changes along the beam path. Hence the discrepancy between the model and the measurement would be larger than 1.6. One of the possible explanations of the discrepancy is a complicated parameter dependence of the index of refraction around a wavelength of $5.3 \mu \mathrm{m}$. As mentioned above, this fitting model ignores the electromagnetic line transitions. There are numerous lines of $\mathrm{H}_{2} \mathrm{O}$ near $5.3 \mu \mathrm{m}$, and the index of refraction shows large resonant characteristic [40]. Additionally, absorption by $\mathrm{H}_{2} \mathrm{O}$ is not 
negligible in that wavelength range [7]. Such local effects might make the dependence on the water vapor density larger.

Validation of the dependence on the temperature is not simple because the temperature changes the relative humidity as well. Ribbon heaters were placed on the optical table, and the temperature was changed by $2.5^{\circ} \mathrm{C}$. As shown in Fig. 5 (b), the relative humidity also changed, while the amount of water vapor was constant. The measured phase shift caused by the temperature change was $-9.0 \mathrm{deg}$. Hence the dependence of $\phi_{0}$ on the temperature is $\Delta \phi_{0}=-0.5$ deg. $/{ }^{\circ} \mathrm{C} / \mathrm{m}$ when the water vapor density is constant at $15.2 \mathrm{~g} / \mathrm{m}^{3}$.

The zero-offset drift observed in the DI on W7-X in Fig. 6 [16] was successfully explained by humidity changes. The similar fitting formula in functions of the humidity, the temperature, and the pressure is used and the fitting parameters are determined to reproduce the measured drifts. The drift is reproduced by the changes in the humidity mainly in the torus hall rather than the temperature and the pressure. This is an evidence that the humidity changes can have a large impact on infrared two-color interferometers, including DIs. This scheme can be implemented as real-time drift compensation [42].

In order to improve the drifts, control of ambient changes, especially the humidity, will be necessary. Since a laser beam path on a fusion plasma device is usually enclosed for laser safety, supply of dry air will be one of the effective and compatible way. Dry air is supplied along the entire beam path, which is about $100 \mathrm{~m}$ long in a far infrared laser interferometer on LHD for the purpose of reduction of laser light absorption by the water vapor [43]. That system successfully reduces the absorption, and has been working for more than 20 years. An enclosure which maintains the water vapor density and the temperature constant can also achieve these results. Another passive way is to use a wavelength located away from the transition lines to reduce of interaction with $\mathrm{H}_{2} \mathrm{O}$. One of the candidates in the $\mathrm{CO}_{2}$ laser is $9.6 \mu \mathrm{m}$. There are few transition lines around the second harmonic $4.8 \mu \mathrm{m}$, different from $5.3 \mu \mathrm{m}$, which is used on LHD and W7$X$. Reduction of the interaction with $\mathrm{H}_{2} \mathrm{O}$ is also required for low-temperature plasmas measurements. In applications to medicine, bodily tissues are directly irradiated, or water is treated with a plasma. Therefore, the plasma always coexists with $\mathrm{H}_{2} \mathrm{O}$. In case of plasma with a liquid anode, the temporal evolution of the measured phase shift suggested changes in the water vapor density [37]. In order to measure the electron density of the plasma under a wet condition, it is preferable to minimize interactions of probe light with $\mathrm{H}_{2} \mathrm{O}$.

\section{Summary}

While a dispersion interferometer (DI) is basically an interferometer, it has prominent advantages over a conventional interferometer: less sensitive to mechanical vibrations and gas density variations. The DI was first applied on a nuclear fusion plasma device in 1990s. Since then, some interferometer techniques (improved homodyne detection, phase modulation, and heterodyne detection) have been introduced and have improved performance. Technical progress in optical components like high efficiency nonlinear crystals also improved the performance of the DIs. In the last two decades, DIs have been installed on several fusion plasma devices and experience in different diagnostic conditions has been gathered. A DI is being designed for ITER to increase the redundancy of the electron density measurement together with the toroidal interferometer polarimeter. In addition to high temperature plasma applications, effectiveness as a low-temperature plasma diagnostic has been experimentally proven. The DI enables the evaluation of the electron density easily, even if the neutral gas density changes substantially. The 
DI can significantly reduce the large phase shift by neutral gas, which usually masks a phase shift by a plasma. The DI is becoming a mature and useful diagnostic to measure electron density of both nuclear fusion and low-temperature plasmas. A remaining challenge is to reduce the perturbations caused by water vapor. On fusion devices, use of dry air is one of the effective ways. Selecting a wavelength away from the $\mathrm{H}_{2} \mathrm{O}$ transition lines which modify the index of refraction of air might be another solution.

\section{Disclaimer}

This report was prepared as an account of work sponsored by an agency of the United States Government. Neither the United States Government nor any agency thereof, nor any of their employees, makes any warranty, express or implied, or assumes any legal liability or responsibility for the accuracy, completeness, or usefulness of any information, apparatus, product, or process disclosed, or represents that its use would not infringe privately owned rights. Reference herein to any specific commercial product, process, or service by trade name, trademark, manufacturer, or otherwise does not necessarily constitute or imply its endorsement, recommendation, or favoring by the United States Government or any agency thereof. The views and opinions of the authors expressed herein do not necessarily state or reflect those of the United States Government or any agency thereof. This study is partially supported by General Atomics Internal Research and Development, Grant-in-Aid for Young Scientists (B) (20760584), Young Scientists (A) (24686104), and the National Institute for Fusion Science grant administrative budget, ULHH027. This work has been also carried out within the framework of the EUROfusion Consortium and has received funding from the Euratom research and training programme 20142018 and 2019-2020 under grant agreement No 633053. The views and opinions expressed herein do not necessarily reflect those of the European Commission.

\section{References}

[1] D. Veron, in Infrared and Millimeter Waves (Academic, New York, 1979), Vol. 2, pp. 67-135

[2] M.A. Van Zeeland et. al., Plasma Phys. Control. Fusion 59, 125005 (2017).

[3] K. Tanaka et. al., Rev. Sci. Instrum 75, 3429 (2004).

[4] H. Lamela et. al., Rev. Sci. Instrum 72, 96 (2001)

[5] Y. Kawano et. al., Rev. Sci. Instrum 67, 1520 (1996)

[6] T.N. Carlstrom et. al., Rev. Sci. Instrum 59, 1063 (1988).

[7] M.A. Van Zeeland et. al., Rev. Sci. Instrum. 89, 10B102 (2018).

[8] V.P. Drachev et. al., Rev. Sci. Instrum. 64, 1010 (1993).

[9] K. Urabe et. al., Appl. Phys. Express 6126101 (2013).

[10] F. Brandi et. al., Rev. Sci. Instrum. 80, 113501 (2009).

[11] P. A. Bagryansky et al., Rev. Sci. Instrum. 77, 053501 (2006).

[12] A. D. Khil'chenko et. al., Instrum. Exp. Tech. 52, 382 (2009). 
[13] T. Akiyama et. al., Rev. Sci. Instrum. 81, 10D501 (2010).

[14] T. Akiyama et. al., Rev. Sci. Instrum. 85, 11D301 (2014).

[15] J. Knauer et. al., Proceeding of 43rd EPS Conference on Plasma Physics, P4.017 (http://ocs.ciemat.es/EPS2016PAP/pdf/P4.017.pdf)

[16] K.J. Brunner et. al., JINST 13 P09002 (2018).

[17] H.X. Wang et. al., Rev. Sci. Instrum. 88, 103502 (2017).

[18] W.M. Li et. al., Rev. Sci. Instrum. 90, 026105 (2019).

[19] T. Wu et. al., Plasma Sci. Technol. 20, 065601 (2018).

[20] T. Akiyama et. al., Rev. Sci. Instrum 74, 2695 (2003).

[21] T. Akiyama et. al., Rev. Sci. Instrum 87, 123502 (2016).

[22] T. Akiyama et. al., Rev. Sci. Instrum 89, 10B105 (2018).

[23] Y. Ito et. al., Fusion Eng. Des., 56-57, 965 (2001).

[24] Y. Jiang et. al., Rev. Sci. Instrum. 68, 902 (1997).

[25] Y. Kawano et. al., Rev. Sci. Instrum. 72, 1068 (2001).

[26] A. Yariv, "Optical Electronics in Modern Communications fifth edition", Oxford University Press.

[27] D. Bamford et. al., Rev Sci Instrum. 84, 093502 (2013).

[28] L.P. Gonzalez et. al., Opt. Lett. 38, 320 (2013).

[29] T. Akiyama et. al. Rev Sci Instrum. 87 11E133 (2016).

[30] D.N. Nikogosyan, "Nonlinear Optical Crystals: A Complete Survey", Springer (2005)

[31] H. Dreier et. al., Rev Sci Instrum. 82, 063509 (2011).

[32] D.J. Campbell et al., J Fusion Energ 38, 11 (2019).

[33] T. Akiyama et. al., JINST 12, C12028 (2017).

[34] H. Sasao et. al., JINST 11, C02082 (2016).

[35] T. Akiyama et. al., Nucl. Fusion 55, 093032 (2015).

[36] K. Urabe et. al., Appl. Phys. Express 6, 126101 (2013).

[37] K. Urabe et. al., Plasma Sources Sci. Technol. 25045004 (2016).

[38] G. Fridman et. al., Plasma Process. Polym. 5, 503 (2008).

[39] R.J. Mathar, J. Opt. A: Pure Appl. Opt. 9, 470 (2007).

[40] R.J. Mathar, Appl. Opt. 43, 928 (2004).

[41] K.J. Brunner et.al., submitted to JINST. 
[42] T. Akiyama et. al., Fusion Sci. Technol. 58, 352 (2010). 